\title{
Desempenho cognitivo em diferentes níveis de escolaridade de adultos e idosos ativos
}

Cognitive performance in different levels of education of adults and active elderly

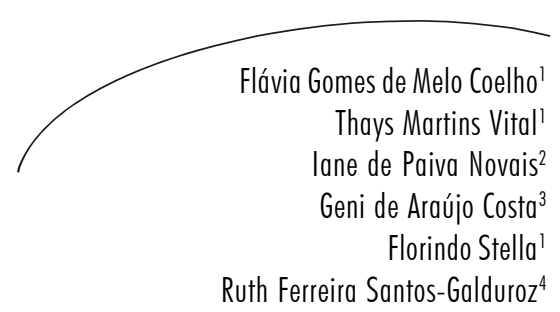

Resumo

Objetivo: Comparar o desempenho cognitivo em diferentes níveis de escolaridade de indivíduos adultos e idosos praticantes de atividade física. Métodos: Foram avaliados 122 indivíduos sem comprometimento cognitivo, idade entre 46 a 85 anos e escolaridade entre 1 a 15 anos, praticantes de atividade física há mais de 6 meses no Programa de Atividades Físicas e Recreativas para a Terceira Idade. Foi aplicada uma bateria de testes cognitivos para verificar as seguintes variáveis: memória de curto prazo, linguagem, aprendizagem, taxa de esquecimento e funções executivas. Resultados: O teste de Kruskal-Wallis apontou diferenças entre os grupos, com diferentes níveis de escolaridade, apenas para a memória de curto prazo, sendo que o teste de U Mann Whitney demonstra diferença entre o grupo de menor escolaridade (1-4 anos) com o grupo de maior escolaridade $(\geq 12$ anos). Conclusão: Diante disso, sugere-se que adultos e idosos ativos, em diferentes níveis de escolaridade, apresentam um perfil cognitivo semelhante para os seguintes domínios cognitivos: linguagem, aprendizagem, taxa de esquecimento e funções executivas.

\section{Abstract}

Objective: To compare the cognitive performance at different education levels of adults and elderly physically active. Methods: The study enrolled 122 individuals without cognitive decline, aged between 46 to 85 years old, 1 to 15 years of schooling. All participants have practiced physical activity for more than 6 months in the Program of Physical and Recreational Activities for the Elderly.

\footnotetext{
Instituto de Biociências, Laboratório de Atividade Física e Envelhecimento. Universidade Estadual Paulista. Rio Claro, SP, Brasil.

2 Instituto de Biociências, Laboratório de Atividade Física e Saúde da Mulher. Universidade Estadual Paulista. Rio Claro, SP, Brasil.

3 Faculdade de Educação Física, Universidade Federal de Uberlândia. Uberlândia, MG, Brasil.

4 Centro de Matemática, Computação e Cognição. Universidade Federal do ABC. Santo André, São Paulo, SP, Brasil.
}

Palavras-chave: Cognição. Atividade motora. Saúde do idoso. Avaliação de desempenho. Atividade física. Nível educacional. 
A battery of cognitive tests was administered to assess the following variables: short-term memory, language, learning, rate of forgetting, and executive functions. Results: The Kruskal-Wallis test showed differences between groups with different levels of schooling, only to the short-term memory, and the Mann Whitney U test shows the difference between the group with less education (1-4 years) with the higher education group ( $\geq 12$ years). Conclusion: Therefore, it is suggested that adults and active elderly, in different levels of schooling, present similar cognitive profile in the following cognitive domains: language, learning, rate of forgetting and executive functions.
Key words: Cognition. Motor activity, Health of the elderly, Employee Performance Appraisal. Physical activity, Education level.

\section{INTRODUÇÃO}

Está bem estabelecido na literatura que o avanço da idade está associado a um declínio no desempenho cognitivo. ${ }^{1}$ As alterações na cognição, quando acompanhadas por outros fatores, podem levar ao surgimento de doenças neurodegenerativas, como a demência de Alzheimer. ${ }^{2}$

Ainda existe dificuldade de identificar e diferenciar o declínio cognitivo não-patológico do declínio cognitivo patológico. Nesse sentido, a avaliação cognitiva constitui um recurso estratégico para a compreensão e seguimento do perfil cognitivo de adultos e idosos. Os testes cognitivos são instrumentos que fornecem dados relativos ao perfil das alterações cognitivas e podem ajudar no diagnóstico precoce de

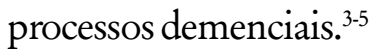

Outro fator muito importante está relacionado à escolaridade, pois o desempenho nos instrumentos de avaliação cognitiva pode ser influenciado pelo nível educacional do indivíduo. Segundo Xavier et al., ${ }^{6}$ idosos com menos tempo de escolaridade e sem demência apresentam pior desempenho em quase todos os testes cognitivos. Em um estudo realizado por Ávila et al., ${ }^{7}$ encontrou-se uma influência da escolaridade na velocidade de processamento, atenção, funções executivas, memória e inteligência, ou seja, os indivíduos com maiores níveis de escolaridade apresentaram melhor desempenho nos testes que avaliam os domínios citados acima. Os autores ainda sugerem que o melhor desempenho pode ser resultado de muitos anos de educação formal, o que torna o cérebro mais resistente e flexível diante dos efeitos de doenças ou das alterações comuns causadas pelo envelhecimento.

Estudos têm verificado que indivíduos que praticam atividade física sistematizada e regular melhoram substancialmente as funções cognitivas. ${ }^{8-10}$ A prática de atividade física interfere de forma positiva na angiogênese, neurogênese e sinaptogênese cerebral através de diferentes mecanismos moleculares. ${ }^{11-12}$ Diante dessa realidade, parece que indivíduos ativos apresentam melhor desempenho cognitivo quando comparados àqueles menos ativos $\mathrm{e}$ hipoteticamente podem apresentar uma relação diferenciada entre escolaridade e função cognitiva.

Em geral, as pesquisas que analisam funções cognitivas são realizadas com idosos atendidos em ambulatórios ou institucionalizados, e os estudos apresentam um consenso em relação ao impacto negativo da escolaridade sobre as funções cognitivas. Por outro lado, são escassos os estudos que avaliam o desempenho cognitivo de idosos e adultos praticantes de atividade física em diferentes níveis de escolaridade. Assim, o objetivo do presente estudo foi comparar o desempenho cognitivo (memória de curto prazo, linguagem, aprendizagem, taxa de esquecimento e funções executivas) em diferentes níveis escolares de adultos e idosos praticantes de atividade física há mais de seis meses.

\section{METODOLOGIA}

\section{Amostra}

Inicialmente, foram convidados todos os indivíduos praticantes de atividade física regular 
há mais de seis meses no Projeto AFRID (Atividades Físicas e Recreativas para a Terceira Idade) da Faculdade de Educação Física da Universidade Federal de Uberlândia. O presente projeto constitui um conjunto de atividades para idosos (acima de 60 anos) e para adultos (acima de 45 anos), e visa a promoção da saúde e melhora da qualidade de vida desses indivíduos.

Após convite, concordaram em participar deste estudo 132 indivíduos. No entanto, dez participantes apresentavam escores no MiniExame do Estado Mental (MEEM) abaixo da média de escolaridade segundo valores sugeridos por Brucki et al..$^{13}$ e por isso foram excluídos do estudo. Os 122 indivíduos que participaram da pesquisa foram considerados saudáveis e preenchiam os seguintes critérios de inclusão: ausência de perda visual e/ou auditiva, ausência de queixas e/ou problemas neurológicos ou psiquiátricos.

O estudo foi aprovado pelo Comitê de Ética em Pesquisa da Universidade Federal de Uberlândia sob o $n^{\circ} .518 / 09$ e cumpriu com os princípios éticos contidos na Declaração de Helsinque. Todos os voluntários assinaram um termo de consentimento livre e esclarecido, que informava os riscos e benefícios da pesquisa.

$\mathrm{Da}$ amostra estudada, a idade variou de 46 a 85 anos, com média de 65,9 anos $( \pm 8,75)$ e mediana de 66 anos. A escolaridade situou-se de um a 15 anos, com média de 6,59 anos $( \pm 4,12)$ e mediana de quatro anos. Os indivíduos foram divididos em grupos de acordo com a escolaridade, do seguinte modo: grupo 1 indivíduos com um a quatro anos de escolaridade, $\mathrm{n}=58(48,7 \%)$; grupo 2 - sujeitos com cinco a oito anos de escolaridade, $\mathrm{n}=28$ (23,5\%); grupo 3 - aqueles com nove a 11 anos de escolaridade, $\mathrm{n}=17(14,2 \%)$ e, finalmente, grupo 4 - indivíduos com escolaridade igual ou superior a 12 anos, $\mathrm{n}=16(13,4 \%)$.

\section{Instrumentos}

Elaborou-se um questionário contendo questões referentes a dados sociodemográficos, idade, gênero e escolaridade. Para a avaliação cognitiva, a equipe recorreu a um conjunto de instrumentos validados, além de serem testes de fácil aplicação e que necessitam de pouco tempo para execução. Os testes foram aplicados na seguinte sequência:

1. Pares Verbais (WMS-R): utilizado para avaliar a aprendizagem e a taxa de esquecimento. O teste é composto por oito pares de palavras, sendo que quatro são considerados pares de associação semanticamente relacionados e quatro não apresentam relacionamento semântico. Inicialmente, o avaliador apresenta verbalmente os oito pares de palavras e imediatamente após o término é apresentada uma palavra por vez, e o participante tentará recordar com as quais se associavam. São realizadas três tentativas e uma última tentativa ocorre após 30 minutos da última exposição aos pares. ${ }^{14}$

\section{Mini-Exame do Estado Mental (MEEM):} é um instrumento composto por questões agrupadas em sete categorias, cada qual planejada com o objetivo de se avaliarem funções cognitivas específicas. São elas: orientação para tempo, orientação para local, registro de três palavras, atenção e cálculo, recordação das três palavras, linguagem e capacidade visuo-construtiva. O escore do MEEM varia de 0 a 30 pontos, e quanto mais baixo o valor, pior está o desempenho cognitivo. ${ }^{15}$

Como o MEEM sofre influência da escolaridade, valores de referência foram propostos com o objetivo de distinguir sujeitos com possíveis déficits cognitivos. Brucki et al. ${ }^{13}$ analisaram uma amostra brasileira e sugeriram os seguintes valores: para analfabetos, 20 pontos; de um a quatro anos de escolaridade, 25; de cinco a oito anos, 26,5 ; de nove a 11 anos, 28; e para indivíduos com escolaridade superior a 11 anos, 29 pontos. Em relação à sensibilidade e especificidade deste instrumento, Bustamante et al. ${ }^{16}$ constataram um índice de sensibilidade de $80 \%$ e de especificidade de $91,3 \%$.

3. Escala Wechsler de Inteligência para Adultos-Revisada / WAIS-III (Wechsler Adult Intelligence Scale-III): foi utilizado o subteste Dígitos composto por recuperação em ordem 
direta e inversa. Este teste avalia memória imediata (ordem direta) e a memória operacional (ordem inversa). ${ }^{17}$

\section{Teste do Desenho do Relógio (TDR):} compreende a tarefa de desenhar um relógio com a inserção dos ponteiros marcando duas horas e 45 minutos. Tem como objetivo avaliar funções executivas (planejamento, sequência lógica, capacidade de abstração e monitoramento de execução), organização visuo-espacial, praxia visuo-construtiva, coordenação psicomotora e memória recente. ${ }^{18}$

\section{Teste de Fluência Verbal Semântica} (TFV): é um instrumento simples, que visa a avaliar o desempenho em geração de palavras e maior número possível da mesma categoria semântica durante um minuto. Este teste avalia velocidade de processamento, memória semântica e principalmente geração de palavras. ${ }^{14}$ Neste estudo foram utilizadas três categorias semânticas: animais, frutas e objetos.
Análise dos dados

A análise estatística foi realizada por meio do programa Statistica, versão 5.0. Devido à natureza escalar das variáveis deste estudo, foram utilizados testes não-paramétricos. Para comparar os grupos, utilizou-se o teste de Kruskal-Wallis, seguido do teste de UMann Whitney, com o ajuste de Bonferroni.

Admitiu-se nível de significância de 5\% $(p<0,05)$.

\section{RESULTADOS}

Na tabela 1, observam-se os escores obtidos no MEEM pelos grupos em diferentes níveis de escolaridade. É importante observar que os participantes não apresentam declínio cognitivo, pois os resultados do estudo estão de acordo com os valores de corte para educação apresentados pelo estudo de Brucki et al. ${ }^{13}$

Tabela 1 - Valor dos escores no MEEM de acordo com os diferentes níveis de escolaridade dos adultos e idosos do Programa de Atividades Físicas e Recreativas para a Terceira Idade/AFRID, de outubro/ 2009 a novembro/2009, Uberlândia, MG. $(\mathrm{N}=122)$

\begin{tabular}{ccc}
\hline Escolaridade & $\mathrm{n}$ & Média \pm DP \\
\hline $1-4$ anos & 61 & $25,56 \pm 2,66$ \\
$5-8$ anos & 28 & $26,14 \pm 2,31$ \\
$9-11$ anos & 17 & $27,06 \pm 0,82$ \\
$\geq 12$ anos & 16 & $28,35 \pm 1,15$ \\
\hline
\end{tabular}

O teste de Kruskal-Wallis não apontou diferenças significativas entre os grupos para os testes de Fluência Verbal, categoria animais e frutas, diferentemente da categoria objetos, na qual o teste de Kruskal-Wallis detectou diferença significativa entre os grupos e o teste de U Mann Whitney mostrou diferença significativa apenas entre o grupo 1 e o grupo 4.
$\mathrm{Na}$ análise descritiva, observa-se que ocorre aumento nos escores médios das categorias animais e frutas, de acordo com o maior nível educacional. Já para categoria objetos, não foi observado o mesmo resultado (tabela 2). 
Tabela 2 - Resultados médios e desvios-padrão nos testes de fluência verbal (categoria: animais, frutas e objetos) de acordo com os diferentes níveis de escolaridade dos adultos e idosos do Programa de Atividades Físicas e Recreativas para a Terceira Idade/AFRID, de outubro/2009 a novembro/2009, Uberlândia, $\mathrm{MG}(\mathrm{N}=122)$.

\begin{tabular}{ccccc}
\hline Escolaridade & $\mathrm{n}$ & $\begin{array}{c}\text { Animais } \\
\text { Média } \pm \mathrm{DP}\end{array}$ & $\begin{array}{c}\text { Frutas } \\
\text { Média } \pm \mathrm{DP}\end{array}$ & $\begin{array}{c}\text { Objetos } \\
\text { Média } \pm \text { DP }\end{array}$ \\
\hline Grupo 1 & 61 & $13,6 \pm 4,17$ & $12,2 \pm 3,03$ & $12,8 \pm 4,65^{*}$ \\
Grupo 2 & 28 & $14,0 \pm 4,03$ & $13,2 \pm 3,61$ & $14,8 \pm 4,76$ \\
Grupo 3 & 17 & $14,2 \pm 3,05$ & $13,4 \pm 2,31$ & $13,0 \pm 3,69$ \\
Grupo 4 & 16 & $17,3 \pm 5,01$ & $14,7 \pm 3,30$ & $17,9 \pm 6,18^{*}$ \\
p & & 0,06 & 0,14 & $0,01^{\S}$ \\
\hline
\end{tabular}

*Diferença significativa entre os grupos 1 e 4 (teste de U Mann Whitney).

`Diferença significativa entre os grupos (teste de Kruskal-Wallis).

No Teste do Desenho do Relógio também não foram observadas diferenças significativas entre os grupos (teste de Kruskal-Wallis). De acordo com a análise descritiva, verifica-se que o desempenho obtido neste teste é maior de acordo com o nível educacional dos indivíduos. Em relação ao teste
Dígitos - ordem direta, o teste de Kruskal-Wallis apontou diferenças significativas entre os grupos, sendo que houve diferença apenas entre os grupos 1 e 4, apontado pelo teste de U Mann Whitney. E para dígitos ordem inversa, não se verificou diferenças entre os grupos.

Tabela 3 - Resultados médios e desvios-padrão no teste do desenho do relógio (TDR) e teste de dígitos na ordem direta (Dig-dir) e na ordem inversa (Dig-inv), de acordo com os diferentes níveis de escolaridade dos adultos e idosos do Programa de Atividades Físicas e Recreativas para a Terceira Idade/AFRID, de outubro/2009 a novembro/2009, Uberlândia, MG. $(\mathrm{N}=122)$

\begin{tabular}{ccccc}
\hline & & TDR & Dig-dir & Dig-inv \\
Escolaridade & $\mathrm{n}$ & Média $\pm \mathrm{DP}$ & Média $\pm \mathrm{DP}$ & Média \pm DP \\
\hline Grupo 1 & 61 & $8,38 \pm 2,07$ & $4,55 \pm 0,87^{*}$ & $3,41 \pm 0,78$ \\
Grupo 2 & 28 & $9,22 \pm 1,05$ & $4,92 \pm 0,81$ & $3,52 \pm 0,96$ \\
Grupo 3 & 17 & $9,5 \pm 0,93$ & $5,0 \pm 0,79$ & $3,25 \pm 0,55$ \\
Grupo 4 & 16 & $9,85 \pm 0,36$ & $5,5 \pm 1,22 *$ & $3,78 \pm 1,12$ \\
p & & 0,06 & $0,01^{\$}$ & 0,27 \\
\hline
\end{tabular}

*Diferença significativa entre os grupos 1 e 4 (teste de U Mann Whitney).

$\checkmark$ Diferença significativa entre os grupos (teste de Kruskal-Wallis).

A figura 1 apresenta os valores médios dos quatro grupos em relação ao teste pares verbais categoria fácil e difícil (tentativa 3), que avalia aprendizagem. O teste de Kruskal-Wallis não indicou diferença significativa entre os grupos. Para investigar a taxa de esquecimento (o número de palavras que o indivíduo esqueceu após 30 minutos), foi realizada a diferença entre a tentativa 4 ea tentativa 3 em ambos os pares verbais. Ao comparar os quatro grupos (teste de Kruskal-Wallis), não houve diferença significativa em relação à taxa de esquecimento para as duas condições (fácil e difícil) (figura 2). 


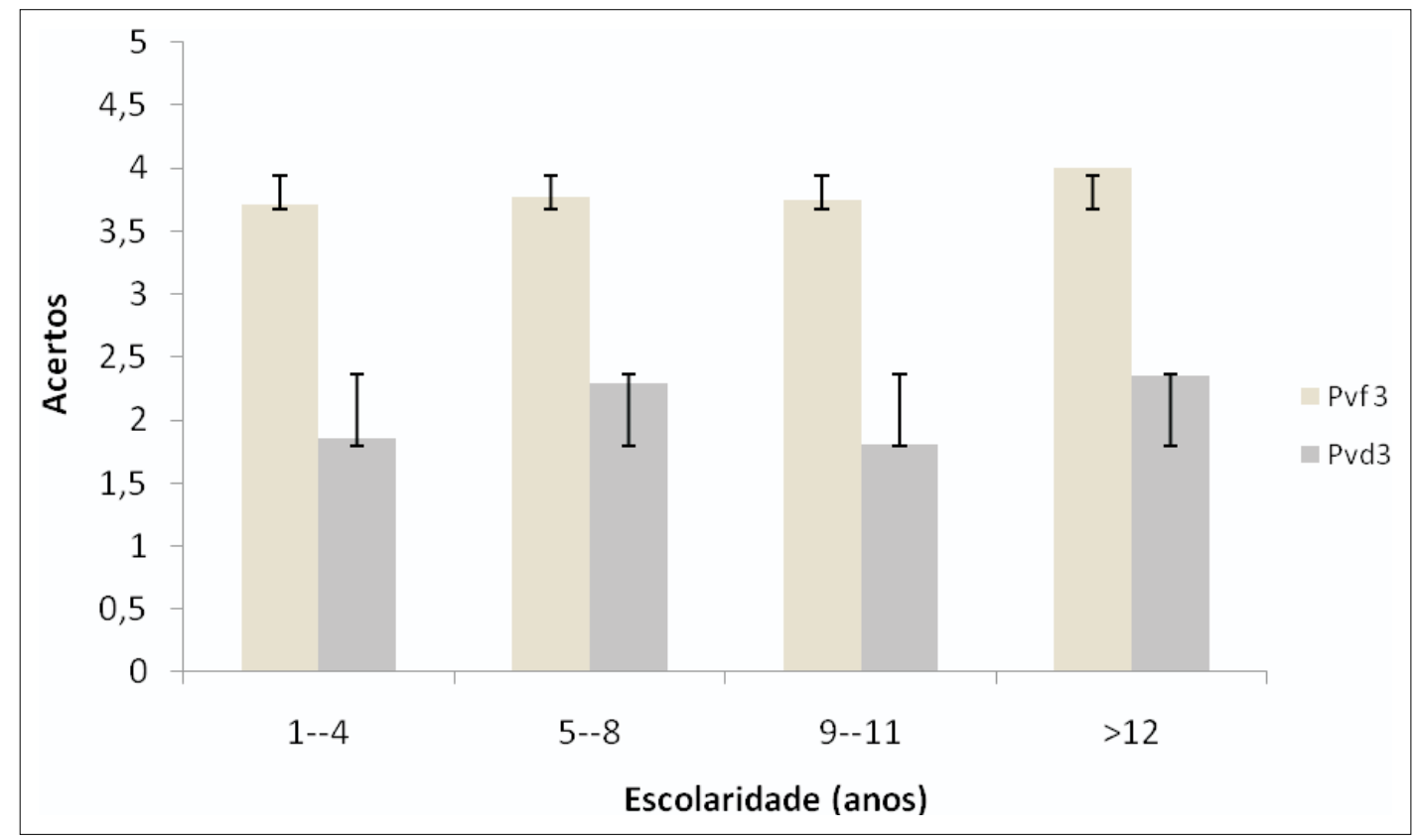

Figura 1 - Valor dos escores no teste pares verbais - categoria fácil (Pvf) e categoria difícil (Pvd) na tentativa 3, de acordo com o nível de escolaridade dos adultos e idosos do Programa de Atividades Físicas e Recreativas para a Terceira Idade/AFRID, de outubro/2009 a novembro/2009, Uberlândia, MG. $(\mathrm{N}=122)$

Pvf- Pares verbais fáceis (Pares semanticamente relacionados);

Pvd- Pares verbais difíceis (Pares sem relacionamento semântico).

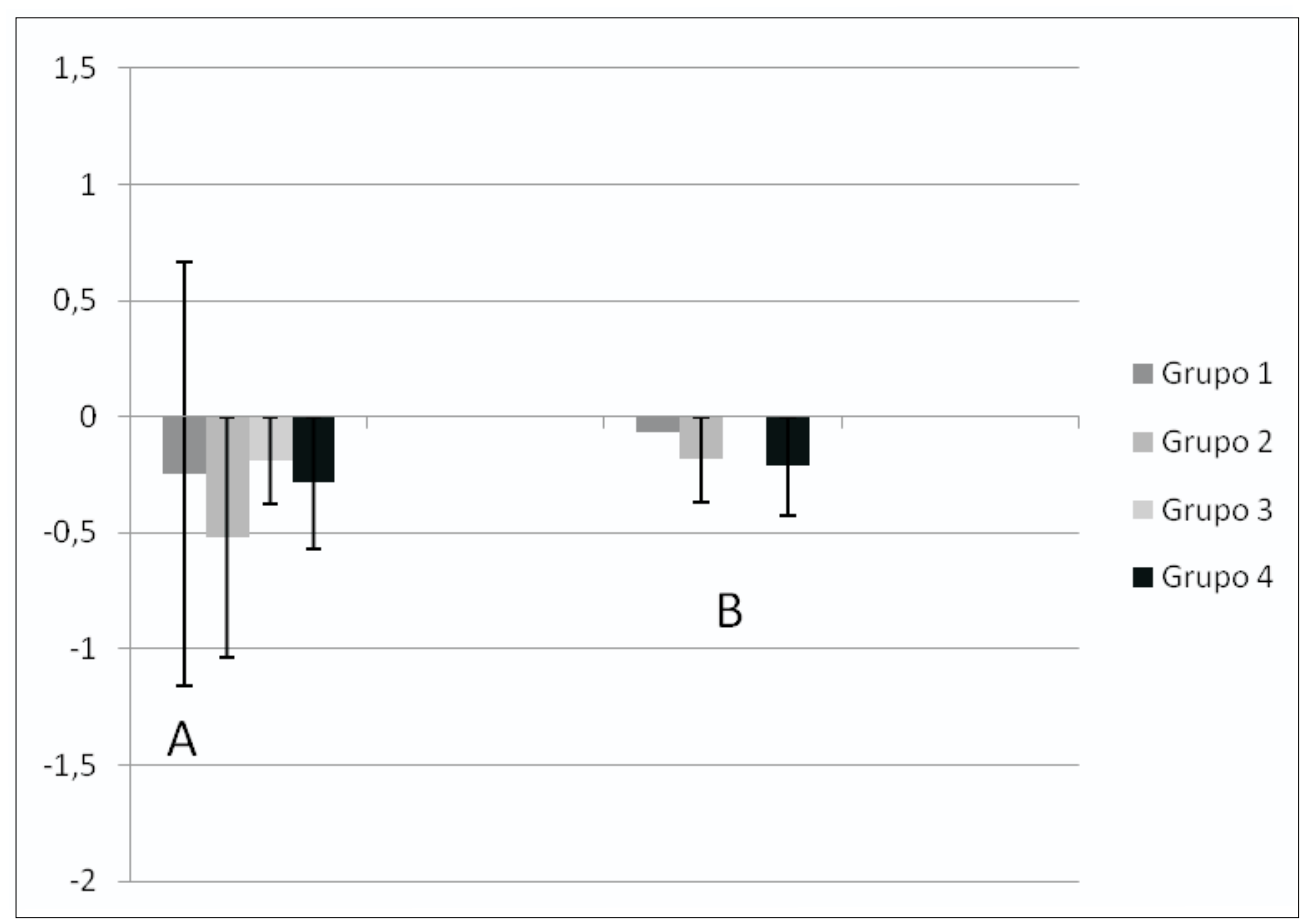

Figura 2 - Taxa de esquecimento para as duas condições - categoria fácil e categoria difícil, de acordo com o nível de escolaridade dos adultos e idosos do Programa de Atividades Físicas e Recreativas para a Terceira Idade/AFRID, de outubro/2009 a novembro/2009, Uberlândia, MG. (N=122). Valores expressos em média \pm desvios padrão.

A - Taxa de esquecimento: Pares semanticamente relacionados (categoria fácil);

B - Taxa de esquecimento: Pares sem relacionamento semântico (categoria difícil). 


\section{DISCUSSÃO}

A preocupação inicial deste estudo foi comparar o desempenho cognitivo de adultos e idosos ativos em diferentes níveis de escolaridade. Verificou-se, a partir dos resultados obtidos, que adultos e idosos ativos, em distintos graus de educação, apresentam linguagem, aprendizagem, taxa de esquecimento e funções executivas similares.

No teste de Fluência Verbal - categoria animais, observa-se que a pontuação no teste aumenta à medida que aumenta o nível educacional. No entanto, não foi encontrada diferença entre os grupos em relação ao desempenho do teste. Ao contrário deste resultado, vários estudos verificaram que a escolaridade interfere no desempenho do teste de Fluência Verbal categoria animais. ${ }^{19-21}$

Na população brasileira, Brucki e Rocha ${ }^{21}$ encontraram interferência da escolaridade na Fluência Verbal - categoria animais. Tal influência persistiu após a divisão dos grupos por nível de escolaridade. A diferença estatisticamente significativa na tarefa de fluência verbal foi observada entre os grupos com menos e mais de oito anos de escolaridade.

No presente estudo, para o teste de Fluência Verbal - categoria frutas, também não se observou diferença entre os grupos com distintos graus de escolaridade. Ao contrário, Yassuda et al. ${ }^{22}$ encontraram diferenças significativas entre os grupos (diferentes níveis de escolaridade) para a tarefa de fluência verbal - categoria frutas.

Os resultados de Brucki e Rocha ${ }^{21}$ e Yassuda et al. ${ }^{22}$ são contrários aos resultados do presente estudo, mas é importante ressaltar que ambos os trabalhos recrutaram suas amostras em um hospital e um Instituto de Psiquiatria. Diferentemente, o presente estudo, avaliou pessoas praticantes de atividade física. Nesse sentido, o estudo de Silva $\&$ Santos $^{23}$ detectou melhor desempenho cognitivo em idosas ativas comparadas com idosas sedentárias e com semelhantes níveis de escolaridade ( 6 anos). Esses autores sugerem que a prática regular de atividade física atuaria como um possível fator de proteção cognitiva e se sobreporia aos efeitos da escolaridade reduzida.

Ainda no presente estudo, a diferença significativa encontrada entre os grupos para o desempenho da tarefa de fluência verbal - categoria objetos foi inconclusivo, pois a pontuação no teste variou entre os diferentes níveis de escolaridade. É importante ressaltar que a sala onde foi aplicada a avaliação cognitiva estava repleta de objetos que eram necessários à mesma, como mesa, cadeira, caneta, lápis, relógio e folhas. Assim, os avaliados, além de dizerem nomes de objetos que lembravam, também diziam os nomes dos objetos que estavam presentes na sala, o que pode ter interferido no desempenho do teste.

Em relação às funções executivas, não foram encontradas diferenças entre os grupos de diferentes níveis de escolaridade para os domínios planejamento e sequenciamento, avaliados pelo Teste do Desenho do Relógio e para a capacidade de manipular mentalmente a informação, avaliados pelo teste Dígitos - ordem inversa. Estudos têm demonstrado melhor desempenho das funções executivas em adultos e idosos praticante de atividade física. ${ }^{24,25} \mathrm{Uma}$ possível hipótese a ser considerada neste trabalho é que o exercício físico pode ter proporcionado benefícios nas funções executivas dos participantes e, diante disso, o desempenho cognitivo foi similar entre os diferentes graus de escolaridade.

Detectou-se, ainda, que a aprendizagem e a evocação tardia (taxa de esquecimento), avaliadas pelo teste Pares Verbais, foram similares entre os grupos de diferentes níveis de escolaridade, o que também pode ter sido melhorado com a prática regular de atividade física. Por outro lado, encontrou-se diferença significativa entre o grupo de menor escolaridade (1-4 anos) com o grupo de maior escolaridade ( $\geq 12$ anos) no desempenho de memória de curto prazo (avaliada através do Teste Dígitos - ordem direta). Uma possível explicação para este achado é que o declínio cognitivo com o avançar da idade está mais relacionado à habilidade de usar a memória de curto prazo enquanto a informação está sendo 
processada $^{26}$ e, neste estudo, o melhor desempenho dos indivíduos na memória de curto prazo pode ter ocorrido por eles serem mais escolarizados e não por praticarem atividade física; ou ainda, talvez o tipo de exercício físico realizado não tenha sido eficaz para melhorar tal domínio cognitivo nos indivíduos.

O presente trabalho detectou que adultos e idosos ativos, em diferentes níveis de escolaridade, apresentam perfil cognitivo similar nos domínios cognitivos, como linguagem, aprendizagem, taxa de esquecimento e funções executivas. No entanto, é fundamental considerar as limitações deste estudo, como a ausência de um grupo de idosos sedentários e um instrumento que analisasse o nível de atividade física dos participantes.

Dados epidemiológicos sugerem que pessoas moderadamente ativas têm menor risco de desenvolver transtornos mentais do que as sedentárias, mostrando que a participação em programas de exercícios físicos exerce benefícios na esfera física e mental. ${ }^{27,28}$ Nesse sentido, é importante ressaltar a hipótese de que o exercício

\section{REFERÊNCIAS}

1. Colcombe SJ, et al. Aerobic fitness reduces brain tissue loss in aging humans. J Gerontol 2003 feb; 58(2): 176-80.

2. Ball LJ, Birge SJ. Prevention of brain aging and dementia. Clinics in Geriatric Medicine 2002 aug; 18(3): 485--503.

3. Bertolucci PHF, et al. Aplicability of the CERAD neuropsychological battery to Brazilian elderly. Arq Neuro-psiquiatria 2001 sept; 59(3A): 532-6.

4. Pawlowski J, Trentini CM, Bandeira DR. Discutindo procedimentos psicométricos a partir da análise de um instrumento de avaliação neuropsicológica breve. Psico-USF 2007 Jul/Dez; 12 (2): 211-9.

5. Angevaren M, et al. Physical activity and enhanced fitness to improve cognitive function in older people without known cognitive impairment. Cochrane Database of Systematic Reviews 2008 Jul; 16(3).

6. Xavier FMF. O transtorno cognitivo da depressão geriátrica ou pseudodemência físico pode ter proporcionado benefícios nas funções cognitivas dos adultos e idosos que participaram deste estudo e, desta forma, a relação entre a escolaridade e a função cognitiva não se manifesta.

\section{CONCLUSÃO}

Os resultados do estudo sugerem que o desempenho cognitivo em adultos e idosos praticantes de atividade física é similar para os diferentes níveis de escolaridade. Recomenda-se, ainda, que futuras investigações comparem idosos ativos versus idosos sedentários, a fim de verificar se a atividade física exerce efeito protetor nas funções cognitivas, independentemente do nível de instrução do indivíduo.

\section{AGRADECIMENTOS}

Programa de Atividades Físicas e Recreativas para a Terceira Idade - AFRID. depressiva do idoso. In: Parente MAMP, et al. Cognição e envelhecimento. Porto Alegre: Editora ArtMed; 2006. p. 203-24.

7. Avila $\mathrm{R}$, et al. Influence of education and depressive symptoms on cognitive function in the elderly. International Psychogeriatrics 2009 jun; 21(3): 560-7.

8. Hillman CH, Erickson KI, Kramer AF. Be smart, exercise your heart: exercise effects on brain and cognition. Neuroscience 2008 Jan; 9(1): 58-65.

9. Colcombe SJ, et al. Neurocognitive aging and cardiovascular fitness: recent findings and future directions. J Molecular Neuroscience 2004; 24(1): 9-14.

10. Kramer AF, Erickson KI, Colcombe SJ. Exercise, cognition, and the aging brain. J Applied Physiology 2006 oct; 101: 1237-42.

11. Lista I, Sorrentino G. Biological mechanisms of physical activity in preventing cognitive decline. Cel Mol Neurobiolo 2010 may; 30(4): 493-503. 
12. Deslandes A, et al. Exercise and mental health: many reasons to move. Neuropsychobiol 2009; 59(4): 191-8.

13. Brucki SMD, et al. Sugestões para o uso do miniexame do estado mental no Brasil. Arq Neuropsiquiatria 2003 sept; 61(3B): 777-81.

14. Lezak MD. Neuropsychological Assessment. 3. ed. New York: Oxford University Press; 1995. $1056 \mathrm{p}$.

15. Folstein MF, Folstein SE, McHugh PR. MiniMental state: a practical method for grading the cognitive state of patients for the clinician. J Psychiatric Research 1975 nov; 12(3): 189-98.

16. Bustamante SEZ, et al. Instrumentos combinados na avaliação de demência em idosos: resultados preliminares. Arq Neuropsiquiatria 2003 sept; 61(3A): 601-6.

17. Wechsler D. WAIS-III: escala de inteligência Wechsler para adultos. In: Silva MCVM . adaptação e padronização de uma amostra brasileira. São Paulo: casa do sociólogo; 2004.

18. Sunderland T, et al. Clock drawing in Alzheimer's disease. A novel measure of dementia severity. J American Geriatrics Society 1989 aug; 37(8): 725-9.

19. Benito-Cuadrado MM, et al. Semantic verbal fluency in animals: a normative and predictive study in a Spanish population. J Clinical and Experimental Neuropsychology 2002 dec; 24(8): 1117-22.

20. Mathuranath PS, et al. Effects of age, education and gender of verbal fluency. J Clinical and Experimental Neuropsychol 2003 dec; 25(8): 1057-64.
21. Brucki SMD, Rocha MSG. Category fluency test: effects of age, gender and education on total scores, clustering and switching in Brazilian Portuguese-speaking subjects. Braz J Medical and Biological Research 2004 dec; 37(12): 1771-7.

22. Yassuda MS, et al. Neuropsychological profile of brazilian older adults with heterogeneous educational backgrounds. Arch Clinical Neuropsychol 2009 feb; 24(1): 71-9.

23. Silva GE, Santos FH. Efeitos do sedentarismo nas funções cognitivas de idosas com escolaridade intermediária. Psico 2009 jan/mar; 40(1): 81-7.

24. Colcombe S, Kramer AF. Fitness effects on the cognitive function of older adults: a metaanalytic study. Psychological Science 2003 Mar; 14(2): 125-30.

25. Hillman CH, Snook EM, Jerome GJ. Acute cardiovascular exercise and executive control function. Int J Psychophysiol 2003 jun; 48(3): 307-14.

26. Binder EF, Storandt M, Birge SJ. The relation between psychometric test performance and physical performance in older adults. The Journals of Gerontol 1999 aug; 54: 428-32.

27. Dustman RE, et al. Aerobic exercise training and improved neuropsychological function of older individuals. Neurobiol Aging 1984 Spring; 5(1): 35-42.

28. Van Boxtel MP, et al. Self-reported physical activity, subjective health, and cognitive performance in older adults. Experimental Aging Research 1996 Oct/dec; 22(4): 363-79. 
